\title{
Avaliação da fluorescência das resinas compostas para esmalte e dentina de diferentes marcas comerciais
}

\section{Evaluation of the fluorescence of enamel and dentin composite resins from different commercial sources}

\author{
Priscilla do Monte Ribeiro Busato ${ }^{1}$, Paola Guellere Saggin ${ }^{1}$, Veridiana Camilotti ${ }^{1}$, \\ Márcio José Mendonça ${ }^{1}$ e Mauro Carlos Agner Busato ${ }^{1}$ \\ ${ }^{1}$ Colegiado de Odontologia, Centro de Ciências Biológicas e da Saúde - CCBS, \\ Universidade Estadual do Oeste do Paraná - UNIOESTE, Cascavel, PR, Brasil \\ *priscillamr@ibest.com.br
}

\begin{abstract}
Resumo
O objetivo do presente estudo foi analisar e avaliar comparativamente pelo método visual, a fluorescência das resinas compostas de cor A2 tanto para esmalte como para dentina, de sete diferentes marcas comerciais. Foram confeccionados para este experimento 52 corpos de prova de resina composta da cor A2 do tipo anterior/posterior, de diversas marcas comerciais disponíveis no mercado. Os corpos de prova foram obtidos com o auxílio de uma matriz de acrílico rígida e transparente. A fotoativação foi realizada com o aparelho 3M Curing Light 2500, com intensidade de $600 \mathrm{~mW} \mathrm{~cm}^{-2}$ pelo período de $40 \mathrm{~s}$. Na superfície dos espécimes foi realizado um polimento com discos Soft-Lex. Foi confeccionada uma caixa de madeira, pintada com tinta preta fosca, para evitar reflexos e a ação de outras fontes de luz. O experimento foi realizado em câmara escura. Nesse experimento três avaliadores calibrados e instruídos avaliaram o grau de fluorescência dos espécimes, quando sob o efeito da iluminação de raios ultravioleta, e os classificaram de acordo com sua intensidade. Para cada avaliador os corpos de prova foram novamente misturados e dispostos aleatoriamente dentro da caixa. Para a avaliação da fluorescência entre resinas de esmalte e dentina, foi aplicado o teste estatístico de Kruskal-Wallis ( $p<0,05)$. Constatou-se que as melhores resinas em termos de fluorescência para esmalte são das marcas comerciais Esthet-X HD e TPH, Opallis e Z350 e para dentina são Opallis e Evolu-X.
\end{abstract}

Palavras-chave: Aluorescência, resinas compostas, características ópticas, estética.

\section{Abstract}

This study analyzes and comparatively evaluate, by a visual method, the fluorescence of the color A2 composite resins for both enamel and dentin from seven different commercial sources. We made fifty-two specimens of anterior/posterior composite resins. The specimens were obtained with a rigid and transparent acrylic matrix. The polymerization was done irradiating with the $3 \mathrm{M}$ Curing Light 2500, intensity of $600 \mathrm{~mW} \mathrm{~cm}^{-2}$ for $40 \mathrm{~s}$. The specimens were polished with Sof-Lex discs. A wooden box painted with flat black paint was used to avoid light reflection and other light sources and the experiment was conducted in a darkroom. Three calibrated and trained evaluators rated the degree of fluorescence of the specimens under the effect of ultraviolet light, and classified them according to their intensity. For each evaluator, the specimens were mixed and randomly arranged inside the box. For the evaluation of fluorescence between resin enamel and dentin, the Kruskal-Wallis test $(\mathrm{p}<0.05)$ was applied. The best resins in terms of fluorescence of enamel are Esthet X-HD, TPH, Z350 and Opallis and for dentin are Opallis and Evolu-X.

Keywords: fluorescence, composites, optical characteristics, esthetic.

\section{Introdução}

As restaurações estéticas nos dias atuais têm como objetivo devolver a estética e função de dentes anteriores e posteriores. Em resultado disso, há a evolução das resinas compostas, nas quais foram feitas modificações físicas e ópticas para melhorar seu desempenho, fazendo com que as restaurações ficassem o mais semelhante possível aos dentes adjacente ${ }^{[1]}$.
Em um dente natural, sua cor é determinada pela interrelação esmalte e dentina através da reflexão e refração da luz. Os materiais restauradores precisariam apresentar propriedades ópticas semelhantes às estruturas dentais, para tornar as restaurações quase que imperceptíveis ${ }^{[2]}$. Entretanto, devido à mudança da iluminação, esses materiais se comportam de formas diferentes. Sendo assim, o cirurgião 
dentista necessita compreender como se manifestam os materiais restauradores e as estruturas dentais sob diferentes fontes de luz ${ }^{[3]}$.

Do ponto de vista estético, a combinação da resistência mecânica, com um bom polimento e características ópticas satisfatórias juntamente com a técnica adequada, são fundamentais para obtenção de excelente resultado ${ }^{[4]}$. No método de transmissão de luz pela estrutura dental, devemos nos atentar para as propriedades que estão diretamente relacionadas com a naturalidade do mesmo, como a transmitância, absorbância e fluorescência apresentada ${ }^{[5]}$.

A transmitância é a quantidade de luz que consegue penetrar e ser transmitida pelo material ou corpo. Devido a essa propriedade, o material desenvolve a aparência de naturalidade à estrutura dental transmitindo-lhe a sensação de profundidade visual à região que recebeu o material, iludindo o observador quanto à autenticidade do mesmo. Já sua propriedade inversa, a absorbância, que tem por função impedir a passagem total de luz pela restauração, com o intuito de minimizar o efeito negativo do fundo enegrecido da boca, uma propriedade importante principalmente em restaurações classe III e IV ${ }^{[5]}$.

A fluorescência é um exemplo de característica óptica relevante nas restaurações estéticas, pois se utilizada uma resina com pouca fluorescência, o dente restaurado ficará mais evidente que os dentes vizinhos, em diversas situações. Em odontologia, a fluorescência pode ser caracterizada como sendo a absorção de luz ultravioleta por uma substância e a emissão posterior dessa luz em forma de espectro azulado ${ }^{[6]}$. Esta fluorescência é policromática e é mais evidente quando os dentes naturais são expostos sob luz negra, como nas discotecas, luz solar, carga das diferentes marcas comerciais assim como a adição de pigmentos. Estes são fatores relevantes para chegar o mais próximo das características ópticas das estruturas dentais ${ }^{[1]}$.

Para que possamos obter a estética desejada, é importante que sejam observadas as características extrínsecas, intrínsecas e ópticas dos dentes naturais, que servem como referência para selecionarmos o material adequado ${ }^{[7]}$.

Devido aos componentes básicos que formam os materiais restauradores não apresentarem propriedades de fluorescência, aparentemente os dentes restaurados com essas resinas compostas apresentam um aspecto mais escuro em relação ao dente natural. Para solucionar este problema, os fabricantes adicionaram agentes luminóforos do grupo metais terras-raras como urópio, térbio, itérbio e cério. $\mathrm{E}$ a incorporação desses pigmentos tem reproduzido satisfatoriamente a fluorescência dos dentes naturais para algumas resinas compostas ${ }^{[4,7]}$.

Um dos fatores importantes na fluorescência presente nos materiais restauradores é devido à forma que emitem luz, fazendo com que pareçam mais brancos e brilhantes, ocasionando em uma aparência de maior vivacidade ${ }^{[8]}$.

Como há grande variabilidade entre as marcas comerciais disponíveis no mercado, frente aos fenômenos ópticos em função das diferentes composições, há uma maior exigência para um resultado estético adequado a cada sorriso.

Os conceitos de materiais restauradores estéticos diretos são relativamente recentes. Por esse motivo, ainda há dúvida com relação à seleção e compra do material ideal para a produção de restaurações estéticas imperceptíveis, capazes de satisfazer o cirurgião dentista e principalmente o paciente ${ }^{[9]}$.

Com base no que foi descrito, esse trabalho tem como objetivo, comparar a fluorescência das resinas compostas tanto para esmalte quanto para dentina de diferentes marcas comerciais.

A hipótese nula do trabalho é de que não haverá diferença estatisticamente significante entre os grupos.

\section{Materiais e Métodos}

Foram confeccionados para este experimento 52 corpos-de-prova de resina composta da cor A2 (de acordo com a escala Vita - Vitapan - Alemanha) do tipo anterior/ posterior, de diversas marcas comerciais disponíveis no mercado, tanto para esmalte quanto para dentina, conforme descrito na Tabela 1. Os corpos-de-prova foram obtidos com o auxílio de uma matriz de acrílico rígida e transparente, em formato circular de $5 \mathrm{~mm}$ de diâmetro e $2 \mathrm{~mm}$ de altura, sendo possível a padronização de todos os espécimes. Essa matriz foi apoiada contra uma placa de vidro e preenchida com um incremento de resina composta para evitar a inclusão de bolhas de ar. Uma tira matriz de poliéster foi aplicada sobre a resina composta e pressionada com outra placa de vidro contra a matriz de plástico rígida, para planificar o espécime, sendo que antes da fotoativação esta placa foi removida. A fotoativação foi realizada com o aparelho $3 \mathrm{M}$ Curing Light 2500 (3M Dental Products), com intensidade de $600 \mathrm{~mW} / \mathrm{cm} 2$ pelo período de 40 segundos, com a ponta ativadora em contato com a tira matriz de poliéster, durante todo o período de fotoativação.

Na superfície dos corpos-de-prova foi realizado desgaste com discos Sof-Lex - (3M ESPE) de grana grossa para remover a camada rica em matriz orgânica evitando o reflexo dessa superfície quando submetida à fonte de raios ultravioleta.

Os corpos-de-prova foram identificados e armazenados individualmente em recipientes de plástico hermeticamente fechados com água, à temperatura de $37^{\circ} \mathrm{C}$, controlada em estufa, pelo período de 7 dias antes da avaliação, onde foram

Tabela 1. Marcas comerciais das resinas compostas testadas.

\begin{tabular}{lll}
\hline & \multicolumn{1}{c}{ Resina - Marca comercial } & \multicolumn{1}{c}{ Cor } \\
\hline G1 & Opallis (FGM- Joiville-SC-Brasil) & A2 esmalte \\
G2 & Opallis (FGM- Joinville-SC-Brasil) & A2 dentina \\
G3 & Z 350 (3M- ESPE- Campinas-SP-Brasil) & A2 esmalte \\
G4 & Z 350 (3M- ESPE- Campinas-SP-Brasil) & A2 dentina \\
G5 & Empress Direct Direct (Ivoclar Vivadent- & A2 esmalte \\
& Barueri-SP-Brasil) & \\
G6 & Empress Direct Direct (Ivoclar Vivadent- & A2 dentina \\
& Barueri-SP Brasil) & \\
G7 & Esthet X HD (Dentsply- EUA) & A2 esmalte \\
G8 & Esthet X HD (Dentsply- EUA) & A2 opaca \\
G9 & TPH (Dentsply- EUA) & A2 esmalte \\
G10 & Amelogen (Ultradent-Indaiatuba-SP-Brasil) & A2 esmalte \\
G11 & Amelogen (Ultradent-Indaiatuba-SP-Brasil) & EW \\
G12 & Evolu-X (Dentsply-EUA) & BW \\
G13 & Evolu-X (Dentsply-EUA) & A2 dentina \\
\hline
\end{tabular}


dispostos aleatoriamente em cartolina preta (Figura 1), antes de serem avaliados.

Foi confeccionada uma caixa de madeira com $22 \mathrm{~cm}$ de largura, $26 \mathrm{~cm}$ de altura e $37 \mathrm{~cm}$ de comprimento, pintada com tinta preta fosca, para evitar reflexos e ação de outras fontes de luz. Pelo mesmo motivo, o experimento foi realizado em câmara escura com os corpos-de-prova iluminados apenas pelas lâmpadas emissoras de radiação ultravioleta. Duas lâmpadas fluorescentes BLB-9W/G23 (90-230V/60Hz) (Masterkey - Tóquio - Japão) com 12,3 cm de comprimento, foram dispostas paralelamente entre si e posicionadas a $16 \mathrm{~cm}$ dos corpos de prova, de modo que a luz ultravioleta fosse emitida perpendicularmente (Figura 2).

Nesse experimento, 3 avaliadores cegos, calibrados e instruídos avaliaram o grau de fluorescência dos corpos-deprova, quando sob o efeito da iluminação de raios ultravioleta,

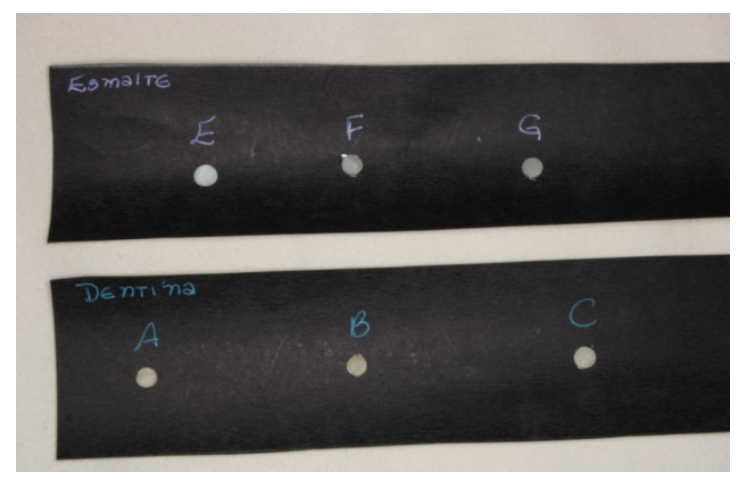

Figura 1. Corpos- de- prova dispostos.

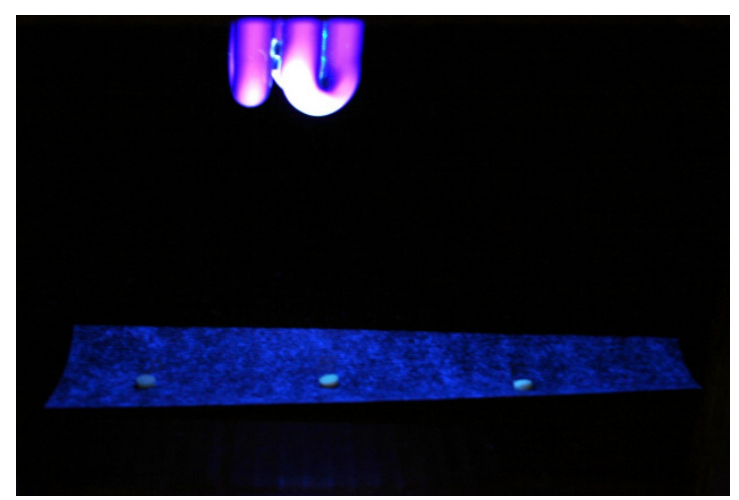

Figura 2. Lâmpada fluorescente posicionada à $16 \mathrm{~cm}$ dos corpos de resina. e os classificaram de acordo com sua intensidade (alta, média, baixa). Com o objetivo de avaliar a concordância entre os examinadores, foi realizado teste estatístico Wilcoxon, não apresentando diferença estatisticamente significante $(\mathrm{p}<0,05)$.

Os corpos-de-prova que emitiram nenhuma ou pouquíssima fluorescência foram classificados como "Baixa Fluorescência", pois apresentam aspecto escuro, recebendo o escore $=1$. Quando os corpos-de-prova emitiram maior fluorescência que os demais apresentando um aspecto mais claro, foram classificados como "Alta Fluorescência" e receberam o escore $=3$. Os demais corpos-de-prova foram classificados como "Média Fluorescência", os que não foram claros nem escuros pela iluminação ultravioleta, receberam o escore $=2$.

Os espécimes foram avaliados uma semana após serem confeccionados, e deixados aleatoriamente dentro da caixa sob o efeito dos raios ultravioleta. A partir daí, os avaliadores iniciaram a separação dos mesmos de acordo com o escore atribuído. Para cada avaliador, os corpos-de-prova foram novamente embaralhados e dispostos aleatoriamente dentro da caixa.

\section{Resultados}

Para a avaliação da fluorescência entre resinas de esmalte e dentina, foi aplicado o teste estatístico de Kruskal-Wallis, onde observou-se diferença estatisticamente significante entre os grupos (Tabela 2), sendo $\mathrm{p}=0,0009$ para esmalte e $\mathrm{p}=0,0001$ para dentina (nível de significância 5\%).

Os grupos G7, G1, G9 e G3 foram os que apresentaram melhores resultados, uma vez que o grau de fluorescência observado foi o de valor mediano, portanto, mais semelhantes à estrutura dentária. Já os grupos G10, G12 e G5 apresentaram alto grau de fluorescência.

Os grupos G11, G4 e G6 demonstraram grau elevado de fluorescência, enquanto o G8 foi o que apresentou grau mais baixo e os restantes, G2 e G13 demonstraram valores medianos.

\section{Discussão}

Houve diferença estatisticamente significante entre os grupos testados, portanto, a hipótese nula do trabalho foi rejeitada.

Marson et al. ${ }^{[2]}$, relataram sobre a importância da habilidade técnica do profissional, juntamente com a sua percepção óptica, pois irá ajudar na criação das restaurações

Tabela 2. Valores médios de fluorescência para resinas de esmalte e dentina.

\begin{tabular}{cccc}
\hline \multicolumn{2}{c}{ Valores médios de fluorescência para resinas de esmalte } & \multicolumn{2}{c}{ Valores médios de fluorescência para resinas de dentina } \\
\hline Grupos & Média (+ou- desvio-padrão) & Grupos & Média (+ou- desvio-padrão) \\
\hline G1 & $2,0(0,0) \mathrm{a}$ & $\mathrm{G} 2$ & $2,2(0,4) \mathrm{ab}$ \\
G3 & $2,2(0,4) \mathrm{a}$ & $\mathrm{G} 4$ & $3,0(0,0) \mathrm{b}$ \\
G5 & $3,0(0,0) \mathrm{b}$ & $\mathrm{G} 6$ & $3,0(0,0) \mathrm{b}$ \\
G7 & $1,8(0,4) \mathrm{a}$ & $\mathrm{G} 8$ & $1,4(0,5) \mathrm{a}$ \\
G9 & $2,0(0,0) \mathrm{a}$ & $\mathrm{G} 11$ & $3,0(0,0) \mathrm{b}$ \\
G10 & $3,0(0,0) \mathrm{b}$ & $\mathrm{G} 13$ & $2,0(0,0) \mathrm{a}$ \\
G12 & $2,4(0,9) \mathrm{ab}$ & $\mathrm{G} 2$ & $2,2(0,4) \mathrm{ab}$ \\
\hline
\end{tabular}

Letras diferentes apresentam diferença estatisticamente significante. 
estéticas. O conhecimento das propriedades ópticas, como a fluorescência dos materiais restauradores é de suma importância nos dias atuais, já que os fatores estéticos são os mais exigidos pelos pacientes que procuram os consultórios odontológicos, a fim de reestabelecer seu sorriso.

De acordo com Pachaly et al. ${ }^{[9]}$, a dentina apresenta baixa translucidez e alta saturação e é a principal responsável pela cor básica e saturação de dentes naturais. O esmalte é significativamente mais translúcido do que a dentina, atenuando sua saturação e agindo como uma fibra ótica capaz de transmitir luz para a dentina subjacente. Neste aspecto, é importante ressaltar que os diferentes graus de fluorescência, interferem significativamente na escolha do material pelo profissional.

Segundo Busato et al. ${ }^{[10]}$, em relação aos dentes posteriores essa diferença é insignificante, já que estas restaurações são mais voltadas para a parte funcional e não para a estética, ao contrário dos dentes anteriores que devem manter as devidas características funcionais, mas principalmente boa estética.

Neste experimento com a metodologia utilizada, foi possível observar que entre as resinas testadas, algumas apresentam alto grau de fluorescência e grande parte delas apresentam valores medianos, e em outras, baixos valores de fluorescências em relação à estrutura dental. As resinas para esmalte das marcas comerciais Esthet-X HD (DentsplyEUA) e TPH (Dentsply- EUA), Opallis (FGM- JoinvilleSC-Brasil) e Z350 (3M- ESPE- Campinas-SP-Brasil) foram as que apresentaram valores entre 1,8 a 2,1 ou seja, valores medianos, já as resinas utilizadas para dentina os valores das marcas Opallis (FGM- Joinville-SC-Brasil) e Evolu-X (Dentsply-EUA) também apresentaram valores médios, consequentemente foram os melhores resultados, pois assemelham-se em maior proporcionalidade com a estrutura dentária. Obtidos estes resultados, ou seja, graus diferentes de fluorescência faz-se necessário considerar a quantidade de resina composta utilizada na estrutura, visto que a menor quantidade de material tende a apresentar grau menor no aspecto fluorescente no dente. Esta situação é extremamente importante diante das exigências estéticas do mercado, principalmente nas estruturas anteriores, em que estas ficam mais expostas. As resinas de elevado grau de fluorescência para esmalte, como as resinas Amelogen (Ultradent-Indaiatuba-SP-Brasil), Evolu-X (Dentsply - EUA) e Empress Direct (Ivoclar Vivadent- Barueri-SP-Brasil), podem ser utilizadas desde que constatada uma estrutura natural remanescente possua um alto grau de fluorescência. Já para dentina foram as marcas Amelogen (UltradentIndaiatuba-SP-Brasil), Z350 (3M- ESPE) e Empress Direct (Ivoclar Vivadent- Barueri-SP-Brasil), que apresentaram alto grau de fluorescência. E apenas a marca comercial Esthet-X (Dentsply - EUA) para dentina foi a que apresentou baixo grau de fluorescência.

Os resultados encontrados neste estudo para a resina Opallis, que se mostrou satisfatória tanto para esmalte quanto para dentina, está de acordo com o que foi encontrado por Jablonski et al. ${ }^{[11]}$, em recente estudo, pois os autores observaram excelentes resultados para essa resina, afirmando que foi a única que mostrou valores de fluorescência semelhantes aos da estrutura dentária ${ }^{[11]}$.
Em outro trabalho que teve como objetivo avaliar a fluorescência para diferentes marcas comerciais, os resultados discordaram dos achados no presente estudo, pois os autores afirmaram que as resinas compostas presentes no mercado odontológico atualmente, apresentam em sua maioria, elevado grau de fluorescência, o que prejudicaria a estética das restaurações em dentes anteriores, enquanto que neste estudo observou-se que a maioria das resinas testadas apresentou valores medianos de fluorescência. Essa diferença de achados pode ser atribuída às marcas comerciais escolhidas nos diferentes trabalhos, uma vez que apresentam diferentes composições ${ }^{[3]}$.

A partir destes resultados podemos observar que os materiais podem apresentar diferenças no grau de fluorescência, o que pode estar relacionado à diferença na composição do material, portanto, são necessários novos trabalhos, que avaliem a propriedade de fluorescência levando em consideração as diferentes marcas comerciais, porém de acordo com sua classificação e composição.

\section{Conclusão}

Dentro das limitações deste estudo e de acordo com a metodologia empregada, constatou-se que as melhores resinas em termos de fluorescência para esmalte são das marcas comerciais Esthet-X HD, TPH, Opallis e Z350 e para dentina são Opallis e Evolu-X.

\section{Referências}

1. Nahsan, F. P. S., Mondelli, R. F. L., Franco, E. B., Naufel, F. S., Ueda, J. K., Schmitt, V. L., \& Baseggio, W. (2012). Clinical strategies for esthetic excellence in anterior tooth restorations: understanding color and composite resin selection. Journal of Applied Oral Science, 20(2), 151-156. http://dx.doi.org/10.1590/ S1678-77572012000200005. PMid:22666829

2. Marson, F. C., Morello, S. R., Michida, S. M. A., Oliveira e Silva, C., \& Correa, G.( 2011). Translucidez e opacidade de compósitos. Revista Científica CRO-RJ, 1(3), 5-9. Retrieved from http://www.cro-rj.org.br/revista/cientifica/revJUL-SET11/ revJUL-SET11.swf

3. Meller, C., \& Klein, C. (2012). Fluorescence properties of commercial composite resin restorative materials in dentistry. Dental Materials Journal, 31(6), 916-923. http://dx.doi. org/10.4012/dmj.2012-079. PMid:23207195

4. Hirata, R. (2008). Avaliação da reflectância, transmitância direta e fluorescência das resinas compostas (Tese de doutorado). Universidade Federal do Rio de Janeiro, Rio de Janeiro.

5. Lefever, D., Mayoral, J. R., Mercade, M., Basilio, J., \& Roig, M. (2010). Optical integration and fluorescence: a comparison among restorative materials with spectrophotometric analysis. Quintessence International, 41(10), 837-844. PMid:20927420

6. Figueiredo, C., Silva, A. M., Figueiredo, A., \& Azenha, M. E. (2012). Fluorescence of dental composite resins. Experimental Pathology and Healthy Sciences, 6(1), 11-14. Retrieved from http://www.patolex.org/revista/revista/201201_files/11-14.pdf

7. Yu, B., \& Lee, Y. K. (2013). Comparison of stabilities in translucency, fluorescence and opalescence of direct and indirect composite resins. European Journal Esthetic of Dentistry, 8(2), 214-225. Retrieved from http://ejed.quintessenz.de/index.ph p?doc $=$ abstract\&abstractID $=29726 /$

8. Takahashi, M. K., Vieira, S., Rached, R. N., Almeida, J. B., Aguiar, M., \& Souza, E. M. (2008). Fluorescence intensity of 
resin composites and dental tissues before and after accelerated aging: a comparative study. Operative Dentistry, 33(2), 189195. http://dx.doi.org/10.2341/07-74. PMid:18435194

9. Pachaly, R., Zasso, M. B., Silveira, M. B., \& Pozzobon, R. T. (2008). Evaluation of Optical Properties of Different Restorative Composite Resins. Revista da Faculdade de Odontologia, Porto Alegre, 49(3), 9-13. Retrieved from http://seer.ufrgs.br/index. php/RevistadaFaculdadeOdontologia/article/view/2805/7633

10. Busato, A. L. S., Reichert, L. A., Valin, R. R., Arossi, G. A., \& Silveira, C. M. (2006). Comparação de fluorescência entre resinas compostas restauradoras e a estrutura dental hígida - in vivo. Revista Odontológica de Araçatuba, 27(2), 142-147. Retrieved from http://apcdaracatuba.com.br/revista/ volume_27_02_2006/PDFs/fluorescencia.pdf

11. Jablonski, T., Takahashi, M. K., Brum, R. T., Rached, R. N., \& Souza, E. M. (2014). Comparative study of the fluorescence intensity of dental composites and human teeth submitted to artificial aging. General Dentistry, 62(1), 37-41. PMid:24401349

Enviado: Dez. 04, 2013 Revisado: Ago. 21, 2014 Aceito: Nov. 17, 2014 